\title{
Identificación de principios de arquitectura empresarial para la gestión de factores de impacto en entidades públicas colombianas utilizando TOGAF
}

\author{
Hernán Cruz Bueno' ${ }^{1}$, Wilson Briceño Pineda ${ }^{22}$
}

Recibido: 2 de marzo de 2015 Aprobado: 25 de mayo de 2015

\begin{abstract}
Resumen
Con el lanzamiento del marco de arquitectura nacional en Colombia, la Arquitectura Empresarial $(\mathrm{AE})$ está tomando cada vez más auge en el sector público, con el propósito de mejorar y transformar la organización desde las Tecnologías de la Información (TI) y el área de negocios. Se han identificado factores de impacto para la implementación de AE que fueron recopilados mediante una revisión bibliométrica de investigaciones internacionales en un trabajo anterior y aunque los principios de arquitectura empresarial (PAE) son considerados como un elemento fundamental en la definición de AE, se encuentran algunas limitaciones en la definición de los mismos, dado que no abarcan y brindan importancia suficiente a dichos factores. Este trabajo propone un conjunto de principios de arquitectura bajo el "framework" de Togaf, basados en la gestión e importancia de los factores de impacto para la implementación de AE en el sector público colombiano, con el fin de convertir a futuro dichos factores en principios y otorgar la importancia requerida a estos, a lo largo de una $\mathrm{AE}$ en una entidad pública.
\end{abstract}

Palabras claves: Arquitectura empresarial, Sector público, Administración pública, Principios arquitectura empresarial.

\section{Abstract}

With the release of national architecture framework in Colombia, Enterprise Architecture (EA) is taking more and more popularity in the public sector, in order to improve and transform the organization from the Information Technology (IT) area Business. There are impact factors identified for the implementation of EA, that were collected through a bibliographical revision of international investigations on previous work and although the principles of enterprise architecture (AEP) are considered as a fundamental element in the definition of EA, there are some limitations on the definition thereof, since these do not cover and give sufficient importance to these factors.

This paper proposes a set of principles of architecture under the "framework" of TOGAF, based on the management and importance of impact factors for the implementation of $A E$ in the Colombian public sector, in order to convert these factors in early and give the importance required to, along a public entity EA.

Keywords: Enterprise architecture, Public sector, Togaf principles, Enterprise architecture principles

\footnotetext{
${ }^{1}$ Magister en Gestión y desarrollo software, Universidad Autónoma de Bucaramanga, Escuela de ingenierías hcruz2@unab.edu.co

2 Magister in Bussines Administration, Southern Illinois University, Carbondale, Decano facultad sistemas UNAB, wbriceno@unab.edu.co. Centro de Investigación en Ingeniería y Organizaciones (CIIO) UNAB.
} 


\section{INTRODUCCIÓN}

La arquitectura empresarial ( $\mathrm{AE})$ es cada vez más reconocida como metodología para la planificación y adaptación de las estrategias, procesos, información, sistemas y tecnologías de una organización y las formas en que estos componentes trabajan juntos con el fin de lograr los objetivos del negocio (Pulkkinen \& Hirvonen, 2005), (Richardson \& Dickson, 1990). Diversas definiciones de AE destacan que los principios de arquitectura empresarial (PAE) son un elemento esencial de cualquier marco de arquitectura empresarial (Esmaeil \& Lewis, 2012). Sin embargo, de acuerdo con estudios sobre PAE (Aier \& Fisher, 2011; Greefhorst \& Proper, 2011; Stelzer, 2010), es necesario profundizar acerca de las limitaciones en la definición y formación de los mismos.

Este trabajo forma parte de una investigación orientada a la identificación, gestión y lineamientos de los factores de impacto sobre la AE para entidades públicas colombianas. Como resultados iniciales de la investigación en grado de maestría, mediante una revisión sistemática de publicaciones en revistas nacionales e internacionales, se identificaron factores de impacto para la implementación de $A E$ en el sector público colombiano (Cruz \& Briceño, 2014). En esta parte de la investigación se busca relacionar el concepto de los principios de arquitectura teniendo en cuenta los factores identificados.

El Ministerio de Tecnologías de la Información y las Comunicaciones (Mintic) lanzó recientemente el marco de referencia para arquitectura empresarial (2014) con el objetivo de unificar en el futuro todas las entidades del Estado con respecto a las tecnologías de información en el ámbito nacional. Sin embargo, no se encuentran estudios a escala nacional que involucren factores de impacto y principios, y cómo están relacionados estos dos en la definición de principios para que exista una trazabilidad de dichos factores a los largo de una $\mathrm{AE}$.

Este artículo se propone revisar y definir principios de $\mathrm{AE}$ con base en el framework Togaf, tras determinar aspectos relevantes para la implementación de $A E$ en el sector público, en busca del impacto de dichos aspectos en proyectos de AE.

\section{MARCO TEÓRICO}

\subsection{Arquitectura empresarial}

Una AE puede definirse como una completa expresión de la organización, que actúa como un plan maestro en colaboración con aspectos de planificación empresarial, operaciones de negocio, automatización, negocios e infraestructura tecnológica (Hirvonen, 2005; Schekkerman, 2005). Para el sector público y los gobiernos locales, la $A E$ puede brindar la vista integral de gestión requerida por éstos, ya que soporta una visión sociotécnica de las organizaciones gubernamentales locales, incluidas las personas, la información, y la tecnología (Saha, 2009; Larsson, 2011). Desde una visión holística de la organización, la $A E$ está representada desde cuatro perspectivas (Saha, 2007; Jin \& Kung, 2010): arquitectura de negocios, arquitectura de aplicaciones, arquitectura de tecnología y arquitectura de datos.

\subsection{Principios de arquitectura}

La fuente principal de los PAE para este estudio es Togaf, que está disponible en el sitio web de Open Group (Togaf, 2009), y deja otros conjuntos de principios para un trabajo futuro. Togaf define como PAE "Las normas y directrices generales, destinadas a ser perdurables y rara vez modificados, que informan y apoyan la forma en que una organización cumple su misión". Togaf señala que los principios de arquitectura se pueden establecer en cuatro grupos (figura 1).

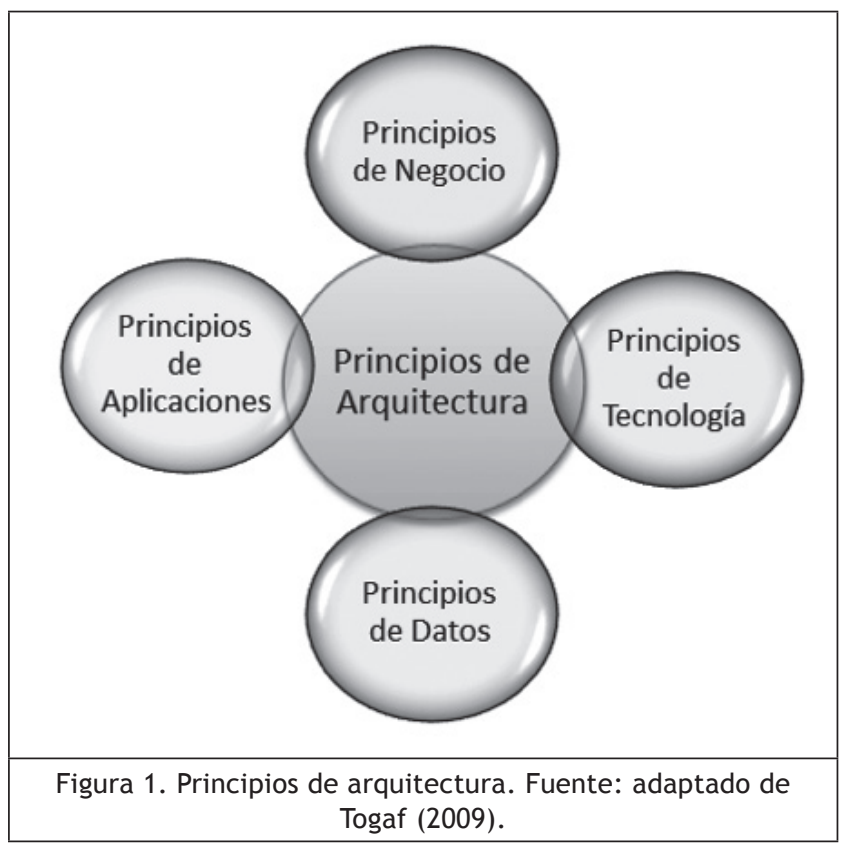

TOGAF define cada PAE en una representación estándar que incluye: nombre, declaración, razón de ser, y las implicaciones. La alineación entre los objetivos de negocio y las capacidades de TI es una clave importante en la definición de los principios en TOGAF.

\subsection{Nodo arquitectura Colombia}

Actualmente el Gobierno nacional posee nodos de innovación, los cuales son espacios de concertación y diseño de soluciones innovadoras a las necesidades y oportunidades TIC identificadas, así como canales de propuesta de proyectos TIC innovadores en temáticas 
estratégicas. Dentro de estos nodos existe uno de innovación en arquitectura TI para gobierno, el cual se contempla como un espacio que facilite la interacción entre entidades oficiales, instituciones académicas y de I+D+i, y empresas alrededor de este tema específicamente, promoviendo la interacción entre los actores, y fomentando el trabajo en equipo y la producción de impactos significativos en el uso y apropiación de TIC en el Gobierno. La arquitectura de tecnologías de información para el Gobierno busca adoptar una colección de modelos de referencia correlacionados que posibiliten el análisis y la identificación de modelos innovadores o de procesos existentes que puedan ser transformados a partir de programas de innovación (Sistema de Investigación, Desarrollo e Innovación, MinTIC, 2012).

\section{METODOLOGÍA}

El diseño general de la investigación constó de cinco fases. En primer lugar, identificación de factores de impacto para proyectos de $\mathrm{AE}$ en el sector público. En segundo lugar, revisión, gestión y diagnóstico de dichos factores por Togaf en su fase preliminar. En tercer lugar, identificación de principios definidos de Togaf que abarcan factores de impacto en sus definiciones. En cuarto lugar, definición y conversión de factores de impacto en principios de arquitectura por tipo, los cuales no son cubiertos por Togaf. En quinto lugar, definición formal de cada principio utilizando Togaf.

\section{RESULTADOS Y DISCUSIÓN}

\subsection{Factores relevantes del sector público}

De acuerdo con los factores identificados por Cruz \& Briceño (2014), se generan tres grupos de factores en tipo social, técnico y administrativo. Dentro de cada uno se muestran los factores de impacto que se deben tener en cuenta al implementar AE en el sector público (tabla 1).

\begin{tabular}{|l|l|l|}
\hline \multicolumn{3}{|c|}{ Tabla 1. Factores de impacto identificados } \\
\hline ASPECTOS SOCIALES & \multicolumn{1}{|c|}{$\begin{array}{c}\text { ASPECTOS } \\
\text { TÉCNICOS }\end{array}$} & $\begin{array}{l}\text { ASPECTOS } \\
\text { ADMINISTRATIVOS }\end{array}$ \\
\hline $\begin{array}{l}\text { Cultura } \\
\text { organizacional }\end{array}$ & $\begin{array}{l}\text { Manejo de } \\
\text { términos }\end{array}$ & $\begin{array}{l}\text { Compromiso } \\
\text { altos mandos }\end{array}$ \\
\hline Comunicación & Interoperabilidad & Presupuesto \\
\hline $\begin{array}{l}\text { Manejo de } \\
\text { involucrados }\end{array}$ & $\begin{array}{l}\text { Seguridad de la } \\
\text { información }\end{array}$ & $\begin{array}{l}\text { Gestión de } \\
\text { riesgos }\end{array}$ \\
\hline $\begin{array}{l}\text { Transformación } \\
\text { organizacional }\end{array}$ & $\begin{array}{l}\text { Herramientas de } \\
\text { AE }\end{array}$ & Visión política \\
\hline $\begin{array}{l}\text { Percepciones } \\
\text { Objetivos }\end{array}$ & $\begin{array}{l}\text { Toma de } \\
\text { decisiones }\end{array}$ \\
\hline Institucionalización & Fuente Cruz \& Briceño (2014) \\
\hline \multicolumn{3}{|c|}{ Gobernanza } \\
\hline
\end{tabular}

Algunos factores influyen de manera considerable en la implementación de arquitectura empresarial
- Cultura organizacional: las estructuras definidas en el tiempo pueden comprometer la aceptación de nuevas tecnologías y procesos.

- Comunicación entre áreas: ya sea entre el arquitecto y los jefes con las áreas involucradas o entre áreas.

- Relación de los involucrados durante las etapas de implementación de $\mathrm{AE}$ en función de su relación y papel dentro de la arquitectura.

- Transformación organizacional, que afecta a toda la entidad.

- Percepción de objetivos: cada persona a cargo puede entender de manera diferente la finalidad y los beneficios de $\mathrm{AE}$.

- Institucionalización de la AE en toda la organización.

- Compromiso de altos mandos a lo largo del proyecto para dar continuidad y seguimiento y buena aceptación en la escala jerárquica.

- Modelo de gobernanza establecido en la organización, con el cual se define cómo mantener la AE y su constante evolución en el tiempo.

- Recursos disponibles en el proceso de implementación.

- Asignación de responsabilidades para toma de decisiones adecuadas.

- Gestión de riesgos en conjunto con AE.

- Visión política.

- Interoperabilidad mediante la utilización de estándares, lenguajes y modelos adecuados.

- Uso apropiado de términos para que cada parte comparta el significado, sobre todo de los de carácter técnico.

- Seguridad de la información.

- Herramientas y software utilizados para el mapeo de la AE teniendo en cuenta tanto aspectos legales como licenciamientos.

En Cruz \& Briceño (2014) se muestra la importancia de tener en cuenta desde el inicio aspectos de tipo social en proyectos de $\mathrm{AE}$ para poder vincular a las personas, elemento clave en cualquier organización desde un enfoque humanístico, dado que los aspectos identificados contribuyen a lograr la sincronización en el uso de las tecnologías de la información y las comunicaciones (TIC) 
y las personas en cualquier organización, y el mayor aprovechamiento sin grandes traumatismos. De igual manera, contar con los aspectos de tipo administrativo permite prever y asumir conflictos de tipo jerárquico, de recursos y políticos que no son ajenos a un proyecto nuevo y en $\mathrm{AE}$ no son la excepción. Se recalca la relevancia e incidencia del manejo administrativo de la organización en los resultados exitosos de una arquitectura empresarial.

Togaf define cuatro conjuntos de principios, por cada grupo estándar aplicable a todo tipo de organización. En este estudio se encuentra que estos principios definidos de forma estándar no dan cobertura o suficiente importancia a aquellos factores de impacto identificados para el sector público, lo cual puede generar una brecha entre la gestión inicial de dichos factores y el ciclo de vida de la arquitectura empresarial. De acuerdo con Togaf, los principios de arquitectura son la base de la $\mathrm{AE}$, por lo cual se propone convertir dichos factores de impacto en principios, con lo cual se asegura la gestión adecuada e importancia en cualquier proyecto de $\mathrm{AE}$ en el sector público y se contribuye a generar mayor probabilidad de éxito en las implementaciones de las mismas.

\subsection{Principios de negocio}

Por defecto, Togaf determina seis principios: maximización de los beneficios para la empresa, gestión de la información, continuidad de negocio, utilización de aplicaciones comunes, orientación al servicio y cumplimiento de la ley y las normas del Estado. En esta investigación se propone ampliar este grupo a 13 (figura 2).

Se encuentra que este grupo es el más impactado con el aumento de principios dado que es el nivel en el que se involucra más lo administrativo y social como parte esencial de los procesos de negocio de una organización. De esta manera se cubren los factores de impacto social y administrativo (tabla 1).

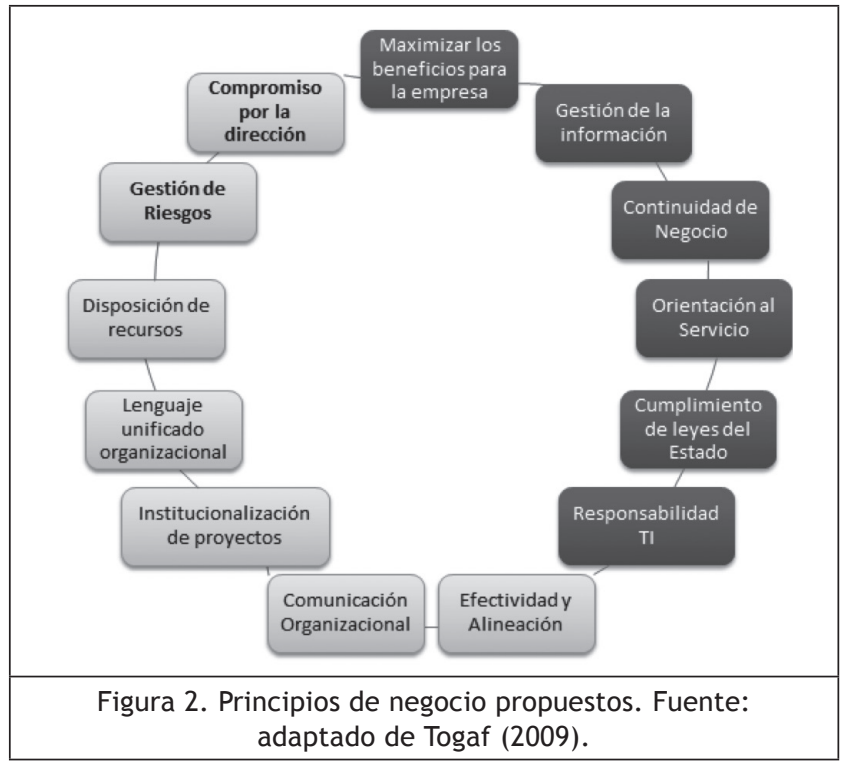

\subsection{Principios de datos}

Por defecto, Togaf plantea cinco principios. En esta investigación se propone ampliar el grupo a seis, agregando un principio de seguridad en la capa de datos (figura 3).

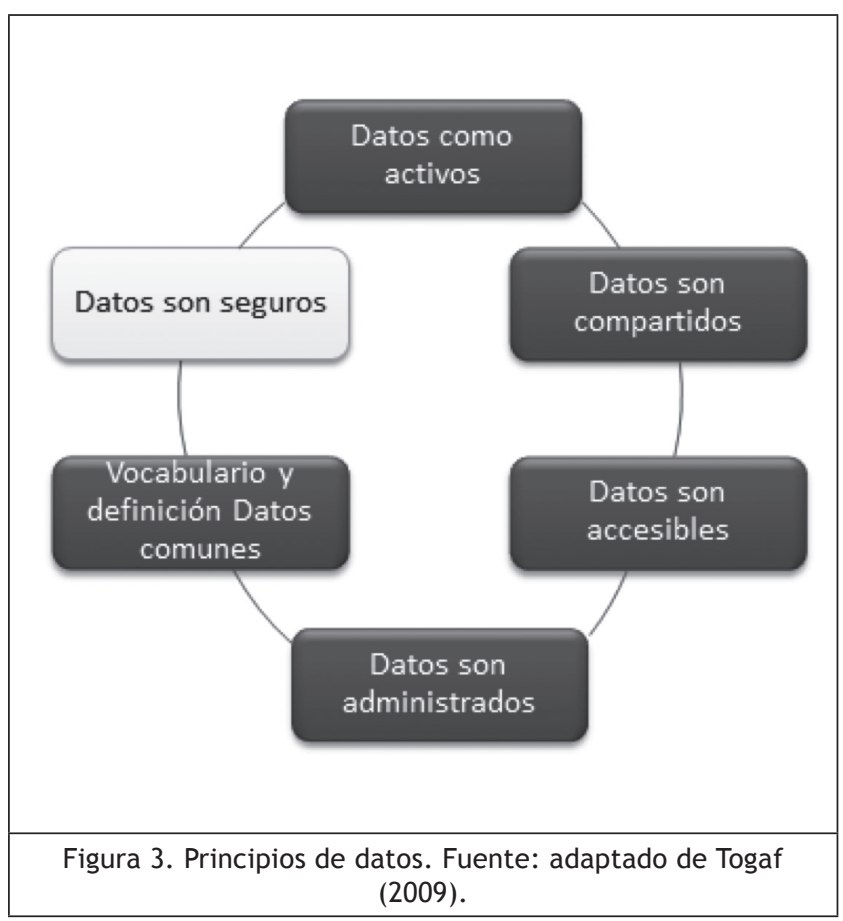

\subsection{Principios de aplicación}

Por defecto, Togaf determina dos principios: independencia de la tecnología y facilidad de uso. En esta investigación se sugiere ampliar el grupo a seis (figura 4).

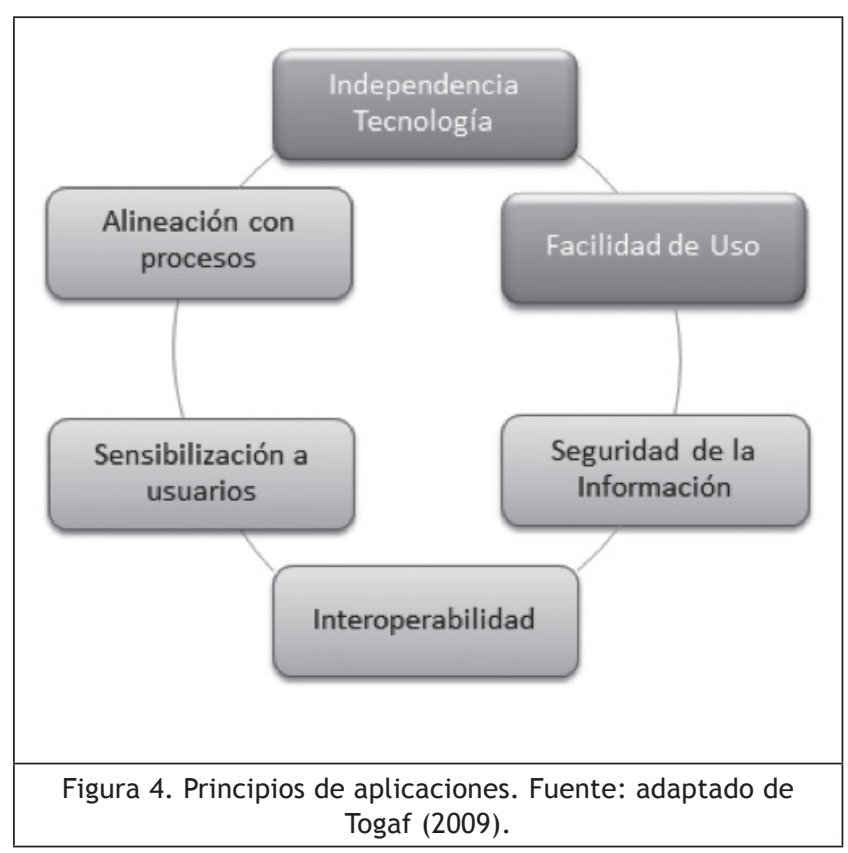


Se proponen estos nuevos principios de aplicación con el fin de gestionar y dar importancia a factores de tipo técnico como seguridad e interoperabilidad; social como los usuarios y su apropiación con aplicaciones, y administrativo con la alineación con procesos y la realidad del negocio que también se presentan a este nivel de aplicaciones.

\subsection{Principios de tecnología}

Por defecto, Togaf propone tres principios y en esta investigación se propone ampliar el grupo a cuatro, añadiendo el principio de calidad de elementos con el fin de garantizar la debida gestión de este aspecto en todo lo relacionado con infraestructura tecnológica (figura 5).

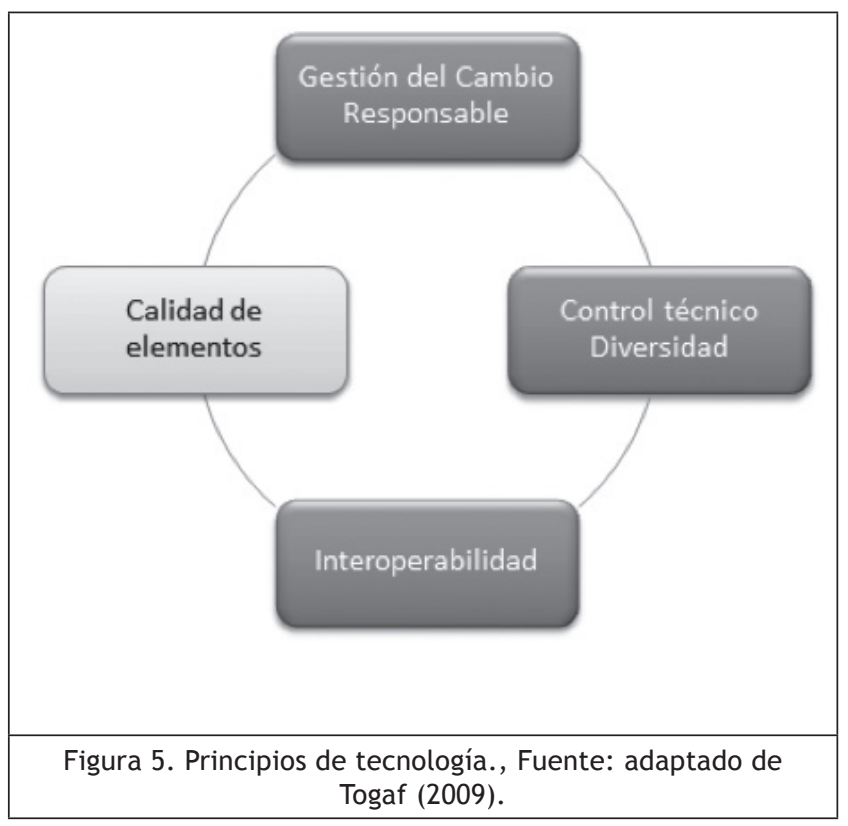

En la investigación realizada se plantea cómo la gestión de factores de impacto en una AE se relaciona directamente con la definición de principios como base principal de arquitectura según el enfoque de Togaf, con el fin de lograr que éstos se tengan en cuenta a lo largo del ciclo de vida de la AE.

En Greefhorst \& Proper (2011), los PAE llenan el vacío entre las intenciones estratégicas de alto nivel y las decisiones necesarias para cumplirlas. Para éste, el concepto de los principios de la arquitectura no han recibido suficiente atención de la investigación. Al mismo tiempo, plantea la necesidad de comprender mejor su esencia. Para Aier \& Fisher (2011), si bien los principios de EA son definidos y documentados, con enfoque en la estrategia de TI, y generalmente percibidos como útiles, las deficiencias se dan en cuanto a los principios de participación de los involucrados, las revisiones periódicas de los mismos, y la alineación de éstos con el negocio y sus componentes.
Hugoson \& Magoulas (2010) argumentan que la elección de los principios para la delimitación y la interoperabilidad tiene un impacto significativo tanto en la alineación operativa como en la estratégica. La elección de un principio arquitectónico adecuado es una condición principal para alinear los sistemas de información a las demandas empresariales y crear beneficios. Pero este autor sólo realizó su estudio desde principios de datos y responsabilidad de TI, con un enfoque más hacia el lado técnico de un proyecto de $A E$. Aun así resalta la importancia de la definición de principios para lograr los resultados esperados.

Togaf (2009) propone un conjunto básico, pero con los resultados de la primera parte de investigación que dio origen a este artículo se sugiere que el grupo se extienda con el fin de definir principios que abarquen factores identificados y gestionados, de alguna manera, en los lineamientos iniciales propuestos. Se plantea darle importancia y definir principios que abarquen comunicación organizacional, institucionalización de proyectos, lenguaje unificado organizacional, disposición de recursos, gestión de riesgos, compromiso por la dirección para la parte de arquitectura de negocios. Para la arquitectura de datos, agregar seguridad de datos. En arquitectura de aplicaciones, tener en cuenta la definición de principios para seguridad de la información, interoperabilidad, sensibilización a usuarios y alineación con procesos. Finalmente, para la arquitectura de tecnología, incluir principios relacionados con gestión de calidad referentes a la parte de adquisición de elementos de infraestructura tecnológica.

\section{CONCLUSIONES Y RECOMENDACIONES}

La fase preliminar de Togaf se puede orientar a entidades públicas teniendo en cuenta los atributos y características particulares de ellas, como organización, lo cual contribuye a reducir la complejidad de su aplicación en proyectos de arquitectura empresarial, teniendo una fase preliminar expresada en términos de revisar el estado actual de una organización compleja, de tipo público, en la cual se definen principios que contemplan factores de impacto, su importancia y gestión.

La definición de principios que reflejan el consenso en toda la empresa y representan el espíritu de la arquitectura empresarial como base de la misma, requiere tener en cuenta factores de impacto para el éxito de $\mathrm{AE}$ con el fin de garantizar que han de estar presentes y con la importancia necesaria durante el ciclo de vida de la arquitectura. Por eso es conveniente convertir estos factores de impacto en principios, como medida de aseguramiento de gestión y control de los mismos. 
Para trabajos futuros se recomienda una exploración más a fondo de los grupos de principios con el fin de evaluar sus estados actuales, priorizar cada principio y su incidencia en la $\mathrm{AE}$, así como los tipos de relación existentes entre ellos.

Recientemente el Gobierno nacional lanzó el marco de referencia para gestión de $\mathrm{TI}$, con el objetivo de unificar las entidades del Estado en materia de TI. Pero no se encuentran investigaciones sobre los efectos, resultados, factores, principios, artefactos y lineamientos por tener en cuenta para iniciar un proyecto de $\mathrm{AE}$ en este sector, de tal manera que los proyectos tengan mayor probabilidad de éxito. Esto es muy importante, dada la tendencia creciente a implementar AE en el sector público. Por tal razón, parte del aporte de esta investigación se enmarca en la definición de PAE para las entidades públicas dentro del marco de arquitectura colombiano, en busca de mediar y prever los grandes impactos negativos que factores identificados previamente puedan tener sobre un proyecto de $\mathrm{AE}$ y la organización durante el ciclo de vida del mismo, ahora que Colombia comienza una promoción de implementaciones de $\mathrm{AE}$ en sus entidades públicas. Aunque entre los hallazgos de la investigación realizada se encuentran y se proponen nuevos principios para cada tipo de arquitectura, es necesario estudiar con detalle el grado de impacto de cada factor de acuerdo con el tipo de entidad pública, ya que esta investigación es un primer acercamiento a factores y principios en entidades públicas para Colombia, pero no se particulariza en ningún tipo de en especial.

\section{REFERENCIAS}

1. Aier, S., Fisher, C. \& Winter, R. (2011). Construction and evaluation of a meta-model for enterprise architecture design principles. Presented at 10th International Conference on Wirtschaftsinformatik, Zurich, Switzerland.

2. Cruz H. \& Briceño W. (2014). Factores relevantes para inicio de arquitecturas empresariales en el sector público colombiano. Estudio bibliométrico. Revista Gerencia Tecnología Informática, UIS, 13(35).

3. Esmaeil, M., Lewis, E. \& Millar, G. (2012) Enterprise architecture principles as values. Journal of Enterprise Architecture, 8(3), pp. 24-34.

4. Greefhorst, D. \& Proper, E. (2011). Architecture principles: the cornerstones of enterprise architecture. Springer-VerlagBerlin and Heidelberg $\mathrm{GmbH} \&$ Co. K.
5. Hirvonen, A. (2005). Enterprise architecture planning in practice. The perspectives of information and communication technology service provider and end-user. Doctoral dissertation, University of Jyväskylä.

6. Hugoson, M. \& Magoulas, T. (2010). Enterprise architecture design principles and business-driven IT management. BIS 2010, 13th International Conference on Business Information Systems, Berlin, Germany, 3-5 de mayo de 2010. LNBIP 57, pp. 144-155. ISBN 978-3-642-15401-0.

7. Jin M. \& Kung D. (2010). Research of Information System Technology Architecture. Second IEEE, International Conference on Industrial and Information Systems, 10-11 de julio de 2010. ISBN:9781424478606.

8. Larsson, H. (2011). Ambiguities in the early stages of public sector enterprise architecture implementation: outlining complexities of interoperability. IFIP - 10th Conference on Electronic Government, EGOV 2011, 28 de agosto a 2 de septiembre de 2011. Springer, ISBN 978-3-642-22877-3.

9. Marco de referencia para gestión de TI en Colombia. 2014 Ministerio TIC. Recuperado de http:// www.mintic.gov.co/gestionti/615/articles-4211_ sumen_del_diseno_y_especificacion_del_Marco_ de_Referencia_de_la_Arquitectura_Empresarial_ para_la_Gestion_TI_del_Estado.pdf.

10. Pulkkinen, M. \& Hirvonen, A. (2005). EA planning, development and management process for agile enterprise development. Proceedings of the 38th Annual Hawaii International Conference on System Sciences, volume 09. ISBN:0-7695-2268-8-9.

11. Richardson, L., Jackson, B. M. \& Dickson, G. (1990). A principle-based enterprise architecture: Lessons From Texaco and Star Enterprise. MIS Quarterly, 14, pp. 385-403.

12. Saha P. (2009). architecting the connected government: practices and innovations in Singapore. The 3rd International Conference on Theory and Practice of Electronic Governance (Icegov 2009), 10-13 de noviembre de 2009. ACM.

13. Saha, P. (2007). Handbook of enterprise systems architecture in practice. IGI Global Information Science Reference, Hershey. ISBN13: 9781599041896.

14. Schekkerman, J. (2005). Enterprise architecture: how are organizations progressing? Web-form Based. 
Institute for Enterprise Architecture Developments. 2005, pp. 79-84.

15. Sistema de Investigación, Desarrollo e Innovación, MinTIC. (2012). Colombia, Documento de plan de acción Nodo de innovación en arquitectura TI para gobierno. Recuperado de http: / / www.mintic.gov. co/portal/604/articles-6305_Agenda_Estrategica_ Nodo_Arquitectura_TI.pdf el 21 de octubre de 2014.
16. Stelzer, D. (2010). Enterprise architecture principles: literature review and research directions. in Service-Oriented Computing. ICSOC/ ServiceWave 2009 Workshops, vol. 6275. A. Dan et al. (eds.). Berlín/Heidelberg: Springer, pp. 12-21.

17. Togaf. (2009). The Open Group Architecture Framework (Togaf). Versión 9, disponible en http://www.opengroup.org/togaf. 